\title{
Analysis of Different Classification Techniques for Two-Class Functional Near-Infrared Spectroscopy-Based Brain-Computer Interface
}

\author{
Noman Naseer, ${ }^{1}$ Nauman Khalid Qureshi, ${ }^{1}$ Farzan Majeed Noori, ${ }^{1}$ and Keum-Shik Hong ${ }^{2}$ \\ ${ }^{1}$ Department of Mechatronics Engineering, Air University, Sector E-9, Islamabad 44000, Pakistan \\ ${ }^{2}$ School of Mechanical Engineering and Department of Cogno-Mechatronics Engineering, Pusan National University, \\ Busan 46241, Republic of Korea
}

Correspondence should be addressed to Noman Naseer; noman@pusan.ac.kr

Received 17 February 2016; Revised 27 May 2016; Accepted 16 June 2016

Academic Editor: Hasan Ayaz

Copyright (C) 2016 Noman Naseer et al. This is an open access article distributed under the Creative Commons Attribution License, which permits unrestricted use, distribution, and reproduction in any medium, provided the original work is properly cited.

\begin{abstract}
We analyse and compare the classification accuracies of six different classifiers for a two-class mental task (mental arithmetic and rest) using functional near-infrared spectroscopy (fNIRS) signals. The signals of the mental arithmetic and rest tasks from the prefrontal cortex region of the brain for seven healthy subjects were acquired using a multichannel continuous-wave imaging system. After removal of the physiological noises, six features were extracted from the oxygenated hemoglobin (HbO) signals. Two- and three-dimensional combinations of those features were used for classification of mental tasks. In the classification, six different modalities, linear discriminant analysis (LDA), quadratic discriminant analysis (QDA), $k$-nearest neighbour $(k N N)$, the Naïve Bayes approach, support vector machine (SVM), and artificial neural networks (ANN), were utilized. With these classifiers, the average classification accuracies among the seven subjects for the 2- and 3-dimensional combinations of features were 71.6, $90.0,69.7,89.8,89.5$, and $91.4 \%$ and $79.6,95.2,64.5,94.8,95.2$, and $96.3 \%$, respectively. ANN showed the maximum classification accuracies: 91.4 and $96.3 \%$. In order to validate the results, a statistical significance test was performed, which confirmed that the $p$ values were statistically significant relative to all of the other classifiers $(p<0.005)$ using HbO signals.
\end{abstract}

\section{Introduction}

Brain-computer interface- (BCI-) based systems provide a direct communication pathway between the brain and external devices without the need for any muscular movements [1]. BCI systems are based on two different approaches, namely, invasive and noninvasive. In invasive BCI systems, for the purpose of fine-quality brain-signal acquisition, electrodes are directly implanted into the brain, which entails high-risk surgery [2-4]. Noninvasive BCI systems, contrastingly, do not require any type of surgery, on which basis they often are preferred over invasive methods. In noninvasive BCI systems, different modalities-electroencephalography (EEG) [5-9], functional magnetic resonance imaging (fMRI) [10-12], and functional near-infrared spectroscopy (fNIRS) [7, 13-21]have been used to acquire high-quality brain signals.

Although fMRI and EEG have shown positive developments for rehabilitation of patients suffering from different motor disabilities, for example, amyotrophic lateral sclerosis (ALS), locked-in syndrome (LIS), and other physical disabilities, fMRI machines are quite expensive as well as heavy, rendering them infeasible for the purposes of portable BCI systems [22]. More recently, alternative fNIRS-based BCI systems have been widely used due to their well-balanced spatial and temporal resolution, safety, ease of use (portability), and less susceptibility to gross electrophysiological artifacts caused by eye blinks, eyeball movements, and muscle activity [23]. Indeed, over the past few decades, fNIRS-based BCI systems have shown promising results in becoming an effective medium of communication for patients with disabilities [18].

Near-infrared spectroscopy (NIRS) functions by utilizing the near-infrared (NI) spectrum of light (wavelength 600 $1000 \mathrm{~nm}$ ) to measure the hemodynamic response represented by oxygenated hemoglobin $(\mathrm{HbO})$ and deoxygenated hemoglobin (HbR), after which the modified Beer-Lambert 
law is used to determine the changes in the $\mathrm{HbO}$ and HbR concentrations $\left(\Delta c_{\mathrm{HbO}}(t)\right.$ and $\Delta c_{\mathrm{HbR}}(t)$, resp.) [24-28]. Jobsis first introduced, in 1977, the principal of near-infrared spectroscopy [29], which entails the use of emitters and detectors separated by a distance of $3 \sim 4 \mathrm{~cm}$. The distance is critical, as a small distance $(1 \mathrm{~cm})$ contains only a skin-layer contribution, while a large distance $(5 \mathrm{~cm})$ can result in lowquality and undesirable signals [23].

In fNIRS-based BCI studies, various mental tasks like motor imagery [15, 16], music imagery [17, 30-32], mental arithmetic (MA) tasks [17, 33, 34], object rotation [34-37], and others [38-41] have been used to acquire maximum classification accuracies that facilitate communication with patients suffering from LIS and ALS. In an fNIRS-based BCI system, the prefrontal cortex of the brain plays an important role in the acquisition of fine signals, for two specific reasons: Usually, it is not involved in motor disabilities, and its hairfree region enhances signal strength and penetration depth [24]. After acquiring brain signals using an fNIRS-based $\mathrm{BCI}$ system, the first step is to eliminate physiological noises using different kinds of filters [42], the next step is to extract the features from the signals, and the final step is to apply classification techniques to acquire the maximum accuracy for the specified task.

In recent decades, various classification schemes have been used in the fNIRS-based BCI area to classify different mental tasks and, thus, acquire maximum classification accuracies, thereby improving the quality and effectiveness of communication with patients suffering afflictions such as ALS and LIS [30, 33, 34, 43-45]. In this study, we acquired mental arithmetic (MA) task versus rest signals from the prefrontal cortex of the brain, after which we removed the signals' physiological noises using the 4th-order Butterworth band-pass filter $[18,19,46]$. Subsequently, those filtered signals were utilized to calculate the different combinations of the statistical properties of the time-domain signals. Then, after obtaining the features, we employed, to acquire maximum classification accuracies across all of the subjects using $\Delta c_{\mathrm{HbO}}(t)$ signals, different types of classifiers, that is, linear discriminant analysis (LDA), quadratic discriminant analysis (QDA), $k$-nearest neighbour $(k N N)$, Naïve Bayes, support vector machine (SVM), and artificial neural networks (ANN). By using 2-dimensional $\Delta c_{\mathrm{HbO}}(t)$ feature combinations with those classifiers, the classification accuracies were $71.6 \pm 1.1,90.0 \pm 1.3,69.7 \pm 0.5,89.8 \pm 1.4,89.5 \pm 1$, and $91.4 \pm$ 0.8 , respectively, and using the 3 -dimensional feature combinations, the classification accuracies were $79.6 \pm 1.5,95.2 \pm 1$, $64.5 \pm 0.3,94.8 \pm 1.2,95.2 \pm 0.7$, and $96.3 \pm 0.3$, respectively.

\section{Materials and Methods}

2.1. Subjects. Seven healthy subjects participated in the experiment. All of them had normal vision and no history of any physical, mental, or psychological disorder. The experiments were conducted in accordance with the latest Declaration of Helsinki, and verbal consent was obtained from all of the subjects after explaining the experimental paradigm.
2.2. Experimental Paradigm. The subjects were seated in a quiet room on a comfortable chair in front of a computer monitor. They were asked to relax and to restrict their motor motions before the start of the experimental paradigm. The subjects were asked to rest and then to perform a mental arithmetic task, as shown in Figure 1(a). Specifically, each subject first rested for $44 \mathrm{~s}$ to adjust the baseline correction of the signals, and then he/she performed a mental arithmetic task for $44 \mathrm{~s}$, of which paradigm was repeated five times. The total length of the experiment was $440 \mathrm{~s}$ for each subject. The $44 \mathrm{~s}$ task-rest periods are rather longer than the conventionally used 20 or 30 s task-rest periods [47-51]. The reason for using a longer duration was to get more data to extract statistical features for the purpose of training the classifiers. Of course, the statistical features are more reliable if the number of data points is larger. Since the main objective of this work was to determine the best performing classifier, training with the reliable and large amount of data was desirable. In the mental arithmetic task, the subjects performed a mental calculation consisting of the subtraction of a two-digit number (10 20) from a three-digit number with successive subtraction of another two-digit number from the result of the initial subtraction (e.g., $300-14,286-11$, and $275-16)[19,43,52]$.

2.3. Optodes Placement. A total of 4 emitters and 10 detectors were positioned on the prefrontal cortex for the detection of mental arithmetic and rest signals, of which configuration included 16 channels. In fNIRS-based BCI systems, the prefrontal cortex is the brain region most widely used, as the hairlessness incurs fewer and less slippage-relatedmotion artifacts and signal attenuation, respectively. The distance between the emitter and the detector plays an important role in the acquisition of fine-quality signals and the obtainment of maximum information therefrom [53]. Usually in fNIRSbased BCI systems, the emitter-to-detector distance is $3 \sim 4 \mathrm{~cm}$ [54]; in our research, the distance was set to $2.8 \mathrm{~cm}$, as shown in Figure 1(b).

2.4. Signal Acquisition. A multichannel continuous-wave system (DYNOT: DYnamic Near-infrared Optical Tomography; two wavelengths: 760 and $830 \mathrm{~nm}$; sampling rate: $1.81 \mathrm{~Hz}$ ) obtained from NIRx Medical Technologies was used for the detection of brain activity. The near-infrared (NIR) light has been transmitted to the scalp from the source with the abovespecified wavelength and then scattered through the cortical region of the brain where chromophores of $\mathrm{HbO}$ and $\mathrm{HbR}$ are present, which absorb some of the NIR light, the rest of which has been detected by the detectors.

2.5. Signal Processing. The modified Beer-Lambert law (MBLL) is used to calculate the concentration changes of $\mathrm{HbO}$ and $\mathrm{HbR}\left(\Delta c_{\mathrm{HbO}}(t)\right.$ and $\left.\Delta c_{\mathrm{HbR}}(t)\right)$ in the microvessels of the cortex:

$$
\begin{aligned}
& {\left[\begin{array}{l}
\Delta c_{\mathrm{HbO}}(t) \\
\Delta c_{\mathrm{HbR}}(t)
\end{array}\right]} \\
& \quad=\frac{1}{l \times d}\left[\begin{array}{ll}
\alpha_{\mathrm{HbO}}\left(\lambda_{1}\right) & \alpha_{\mathrm{HbR}}\left(\lambda_{1}\right) \\
\alpha_{\mathrm{HbO}}\left(\lambda_{2}\right) & \alpha_{\mathrm{HbR}}\left(\lambda_{2}\right)
\end{array}\right]^{-1}\left[\begin{array}{l}
\Delta A\left(t, \lambda_{1}\right) \\
\Delta A\left(t, \lambda_{2}\right)
\end{array}\right],
\end{aligned}
$$




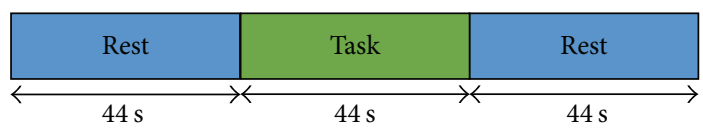

(a)

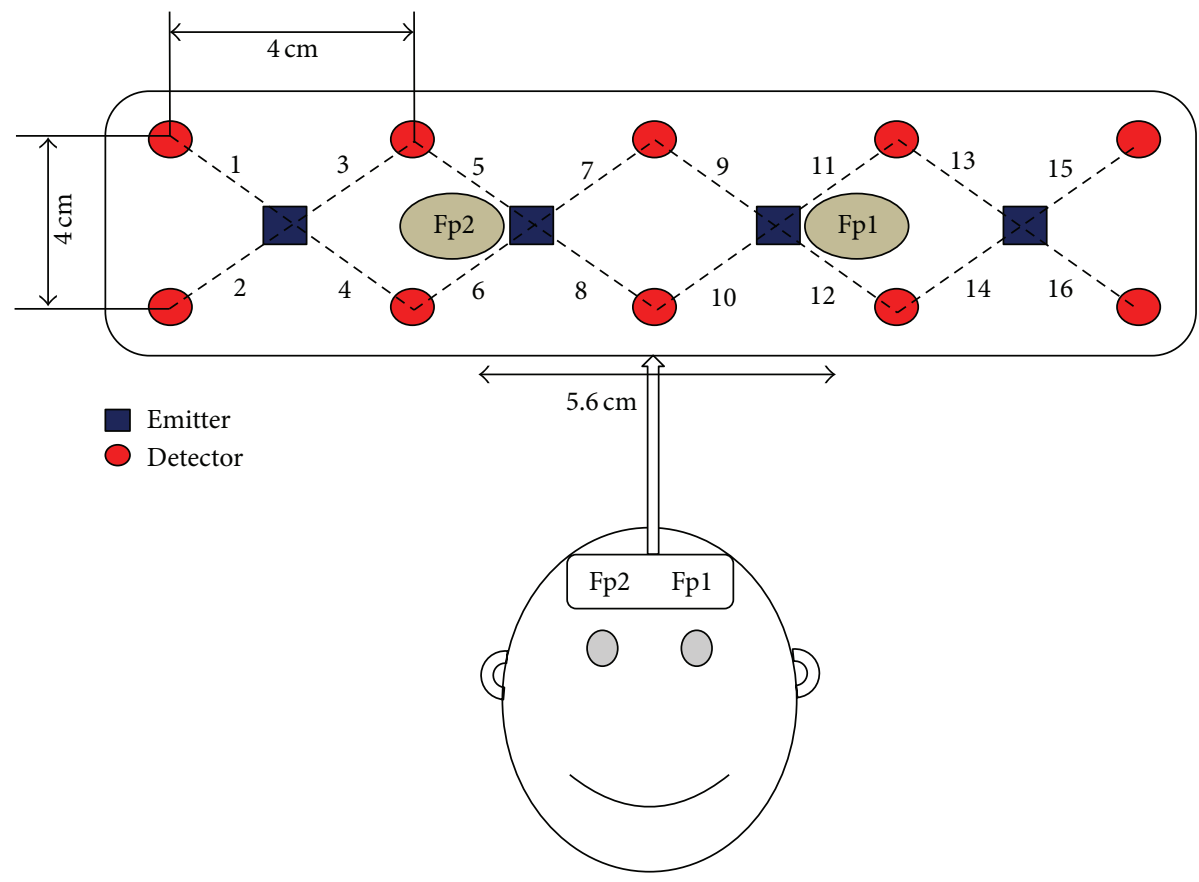

(b)

Figure 1: (a) Schematic of the experimental paradigm: the blue blocks represent the $44 \mathrm{~s}$ rest periods at the beginning and at the end; the second, green block represents the $44 \mathrm{~s}$ mental arithmetic task; (b) optode placement and channel location on the prefrontal cortex. Fpl and Fp2 are the reference points of the international 10-20 system.

where $\Delta A\left(t ; \lambda_{j}\right)(j=1,2)$ is the absorbance (optical density) measured at two points of wavelength $\lambda_{j}, a_{\mathrm{HbX}}\left(\lambda_{j}\right)$ is the extinction coefficient of $\mathrm{HbX}$ (i.e., $\mathrm{HbO}$ and $\mathrm{HbR}$ ) in $\mu \mathrm{M}^{-1} \mathrm{~mm}^{-1}, d$ is the differential path length factor (DPF), and $l$ is the emitter-detector distance (in millimetres). The signals obtained after conversion to $\Delta c_{\mathrm{HbX}}(t)$ contain physiological noises; so, we used a notch filter with band-reject ranges of $1 \sim 1.2 \mathrm{~Hz}, 0.3 \sim 0.4 \mathrm{~Hz}$, and below $0.01 \mathrm{~Hz}$ to minimize the effects of such heartbeat-, respiration-, and Mayer-waverelated noises, respectively.

2.6. Feature Extraction. In this study, we used the following statistical properties of time-domain signals as features: signal mean $[18,36,45,52,55,56]$, signal peak $[33,45,57]$, signal slope [18, 58], signal variance [45, 59], signal kurtosis [45, 59], and signal skewness $[45,59]$. Two- and three-dimensional combinations of those features were used for classification of the signals extracted from $\Delta c_{\mathrm{HbO}}(t)$. These features were calculated across all 16 channels spatially during the entire task and rest periods. All the features were normalized between 0 and 1 by the following equation [42]:

$$
x^{\prime}=\frac{x-\min (x)}{\max (x)-\min (x)},
$$

where $x^{\prime}$ represents the feature values rescaled between 0 and $1, x \in R^{n}$ are the original values of the features, and $\max (x)$ and $\min (x)$ represent the largest and smallest values, respectively. Figure 2 shows the $3 \mathrm{D}$ feature space of the mental arithmetic and rest tasks for mean, speak, and skewness.

\subsection{Classification}

2.7.1. Linear Discriminant Analysis. LDA has been most frequently used for pattern recognition in fNIRS-based BCI systems, thanks to its low computational cost and high speed $[46,55,60-62]$. Basically, LDA finds the projection to a line such that the samples from the classes are well separated from each other, thus achieving its main objective, dimensionality reduction. LDA does this, specifically, by maximizing the ratio of between-class variance and minimizing the ratio of within-class variance. The Matlab ${ }^{\circledR}$ command "classify linear" was used with 10-fold cross-validation to extract the classification performance.

2.7.2. Quadratic Discriminant Analysis. QDA, likewise, maximizes the ratio of between-class variance and minimizes the ratio of within-class variance; however, it also allows 


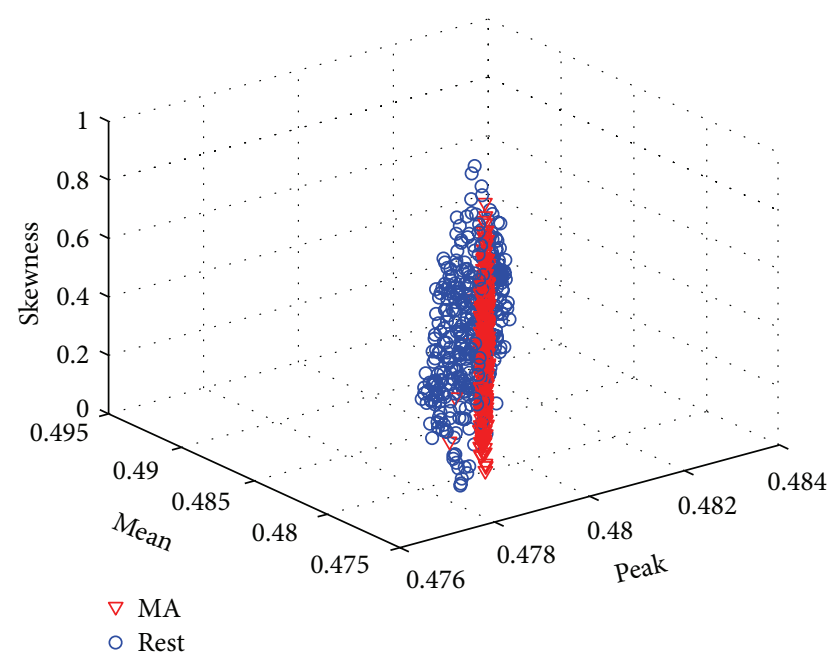

FIGURE 2: 3D scatter plot of the signal mean, signal peak, and signal skewness values of $\mathrm{HbO}$ (subject 2).

quadratic decision boundaries between classes, thereby enabling the classifier to perform more effectively and enhancing classification accuracy $[17,63]$. The Matlab ${ }^{\circledR}$ command "classify quadratic" was used with 10 -fold crossvalidation to extract the classification performance. In the present work, normal LDA and QDA, that is, without shrinkage or regularization, are used.

2.7.3. $k$-Nearest Neighbour. $k \mathrm{NN}$ is the simplest classification technique used in fNIRS-based BCI systems for machinelearning algorithms [64]. The $k \mathrm{NN}$ algorithm works by determining which of the points from the training data are close enough to be considered when selecting the class to predict for a new observation. In the present research, the value of $k$ was set to 1 in order to allow for the closest training samples of the class. The Matlab ${ }^{\circledR}$ command " $k \mathrm{NN}$ classify" was used with 10 -fold cross-validation to extract the classification performance.

2.7.4. Naïve Bayes Classifier. In addition to LDA, QDA, and $k \mathrm{NN}$, the Naïve Bayes approach was also implemented in our study, due to its simplicity and transparency in machinelearning modalities. This approach is fundamentally based on the Bayes theorem with assumptions of strong independence among the features $[65,66]$ :

$$
P(c \mid x)=\frac{P(x \mid c) P(c)}{P(x)}
$$

where $P(c \mid x)$ is the feature probability of the class (target) of a given feature, $P(c)$ is the prior probability of the class, $P(x \mid c)$ is the likelihood which is the probability of feature given class, and $P(x)$ is the prior probability of the feature.

2.7.5. Support Vector Machine. SVM is a widely employed classification modality in fNIRS-based BCI systems due to its high classification performance, relatively good scalability to high-dimensional data, and explicit control of errors [19, $34,44,59,67,68]$. The main idea of SVM is to create the hyperplanes that maximize the margins between the classes that can be obtained by minimizing the cost function and, thereby, enable maximum classification accuracy. The vectors that represent the hyperplanes are known as support vectors. The optimal solution $r^{*}$ that maximizes the distance between the hyperplane and the nearest training point(s) can be obtained by minimizing the cost function:

$$
\begin{array}{ll}
\text { Minimize } & \frac{1}{2}\|w\|^{2}+C \sum_{i=1}^{n} \xi_{i} \\
\text { Subject to } & y_{i}\left(w^{T} x_{i}+b\right)^{3} \geq 1-\xi_{i}, \quad \xi_{i} \geq 0,
\end{array}
$$

where $w^{T}, x_{i} \in R^{2}$ and $b \in R^{1},\|w\|^{2}=w^{T} w, C$ is the tradeoff parameter between error and margin, $\xi_{i}$ is the measure of training data, and $y_{i}$ is the class label for the $i$ th sample. The main advantage of SVM is that it can be used as both a linear and a nonlinear classifier. In order to make SVM a nonlinear classifier, one of various types of kernel functions (i.e., polynomial, radial basis, and sigmoid functions) can be used. In our present research, we utilized a third-degree polynomial kernel function with $C=0.5$. Tenfold cross-validation was then used to estimate the classification accuracies. The reason for using nonlinear SVM is that it has been shown to yield better classification accuracies than the linear classifiers [19].

2.7.6. Artificial Neural Networks. ANN is a classification technique widely used for deep machine-learning and pattern recognition in fNIRS-based BCI system $[35,69,70]$. The ANN classification modality plays an important role in the rehabilitation of patients suffering from afflictions such as ALS and LIS by decoding useful information. In our research, we used a three-layer perceptron consisting of an input, a hidden layer, and an output. The numbers of hidden neurons are specified by the following equation:

$$
H=[(N+M) d],
$$

where $N$ is the number of input neurons, $M$ is the number of output neurons, and $d$ is a constant with $d \in(0,1]$. For ANN classifier, the Matlab toolbox was used with 10 hidden neurons, $70 \%$ of the total data was used for training, $15 \%$ data was used for validation (measure of network generalization), and $15 \%$ data was used for testing (independent measure of network performance during and after training) [71].

\section{Results and Discussion}

In this study, we analyse and compare the performance of LDA, QDA, $k \mathrm{NN}$, Naïve Bayes, SVM, and ANN classifiers in order to determine the best classifier for fNIRS-based BCI system using mental arithmetic tasks and rest. The classification accuracies for mental arithmetic task and rest were calculated for all possible 2- and 3-feature combinations of six different features. The extracted features include the 
TABLE 1: Classification accuracy using LDA among all subjects.

\begin{tabular}{|c|c|c|c|c|c|c|c|}
\hline Feature combination & S1 & $\mathrm{S} 2$ & S3 & $\mathrm{S} 4$ & S5 & S6 & S7 \\
\hline Mean \& slope & 53.2 & 49.2 & 50.1 & 58.3 & 59.8 & 55.8 & 59.6 \\
\hline Mean \& peak & 94.5 & 96.7 & 90.3 & 92.0 & 91.1 & 92.2 & 94.9 \\
\hline Mean \& variance & 86.8 & 87.6 & 81.7 & 82.9 & 82.8 & 76.4 & 83.4 \\
\hline Slope \& peak & 87.3 & 83.6 & 80.8 & 85.9 & 83.8 & 83.6 & 81.2 \\
\hline Slope \& variance & 87.5 & 88.3 & 83.2 & 82.6 & 81.5 & 76.4 & 79.9 \\
\hline Peak \& variance & 89.7 & 89.8 & 83.7 & 87.5 & 87.3 & 83.7 & 81.2 \\
\hline Peak \& skewness & 89.1 & 83.6 & 80.4 & 86.5 & 81.6 & 83.2 & 81.2 \\
\hline Mean \& skewness & 49.6 & 50.6 & 48.6 & 53.3 & 53.6 & 53.5 & 50.1 \\
\hline Slope \& skewness & 50.5 & 51.2 & 50.3 & 53.8 & 54.0 & 53.1 & 50.9 \\
\hline Kurtosis \& skewness & 47.7 & 51.2 & 50.6 & 53.2 & 50.4 & 53.8 & 51.6 \\
\hline Variance \& skewness & 88.0 & 89.0 & 82.3 & 83.4 & 81.4 & 78.2 & 84.4 \\
\hline Peak \& kurtosis & 86.8 & 82.4 & 80.9 & 85.9 & 83.9 & 82.6 & 81.2 \\
\hline Mean \& kurtosis & 47.4 & 49.7 & 52.2 & 54.8 & 50.4 & 52.1 & 48.6 \\
\hline Slope \& kurtosis & 45.7 & 46.2 & 54.3 & 54.6 & 52.1 & 50.1 & 47.7 \\
\hline Variance \& kurtosis & 87.6 & 88.5 & 82.1 & 83.2 & 82.4 & 82.2 & 86.3 \\
\hline
\end{tabular}

TABLE 2: Classification accuracy using QDA among all subjects.

\begin{tabular}{lccccccc}
\hline Feature combination & S1 & S2 & S3 & S4 & S5 & S6 \\
\hline Mean \& slope & 95.5 & 96.5 & 95.5 & 96.6 & 96.0 & 95.4 & 96.9 \\
Mean \& peak & 97.0 & 98.4 & 97.7 & 98.2 & 97.4 & 98.4 & 98.4 \\
Mean \& variance & 97.4 & 98.0 & 96.0 & 96.5 & 96.1 & 95.4 \\
Slope \& peak & 94.4 & 95.0 & 88.5 & 95.7 & 95.1 & 93.6 & 97.2 \\
Slope \& variance & 98.0 & 98.2 & 94.0 & 95.5 & 94.4 & 93.0 & 95.1 \\
Peak \& variance & 97.0 & 98.1 & 93.7 & 97.4 & 96.0 & 94.1 \\
Peak \& skewness & 90.6 & 89.8 & 83.8 & 92.6 & 89.7 & 88.8 \\
Mean \& skewness & 91.0 & 91.5 & 90.5 & 91.8 & 89.1 & 87.6 \\
Slope \& skewness & 89.3 & 89.7 & 84.2 & 88.0 & 90.0 & 86.6 \\
Kurtosis \& skewness & 48.3 & 53.7 & 51.4 & 52.8 & 50.2 & 96.8 \\
Variance \& skewness & 97.5 & 97.9 & 90.7 & 95.6 & 94.7 & 96.1 & 90.7 \\
Peak \& kurtosis & 89.6 & 88.2 & 82.7 & 92.1 & 88.1 & 88.2 \\
Mean \& kurtosis & 89.2 & 91.1 & 88.8 & 90.8 & 88.8 & 82.1 \\
Slope \& kurtosis & 89.5 & 89.6 & 84.3 & 87.7 & 89.8 & 87.5 \\
Variance \& kurtosis & 97.6 & 97.9 & 90.6 & 95.4 & 94.4 & 86.6 & 90.6 \\
\hline
\end{tabular}

signal mean, signal peak, signal skewness, signal slope, signal variance, and signal kurtosis. These features are calculated for the whole task and rest periods. It was found that the presence of signal mean and signal peak in both 2- and 3-feature combinations yielded maximum classification accuracies. This finding is an endorsement to our previous finding in [21].

Tables 1, 2, 3, 4, 5, and 6 show the classification accuracies among all of the subjects for the respective classifiers. Those accuracies were extracted from 2-dimensional combinations of features derived from $\Delta c_{\mathrm{HbO}}(t)$ signals. The average classification accuracies of the LDA, QDA, $k N N$, Naïve Bayes, SVM, and ANN classifiers for the 2-dimensional feature combinations were $71.6 \pm 1.1,90.0 \pm 1.3,69.7 \pm 0.5,89.8 \pm$ $1.4,89.5 \pm 1$, and $91.4 \pm 0.8$, respectively. To further examine the performances of the classifiers used in our study, we also employed 3-dimensional combinations of features and extracted the corresponding classification accuracies, which were $79.6 \pm 1.5,95.2 \pm 1.0,64.5 \pm 0.3,94.8 \pm 1.2,95.2 \pm 0.7$, and $96.3 \pm 0.3$, respectively. In both (2- and 3-dimensional) cases, it was found that the ANN classifier has the highest classification accuracies: 91.4 and $96.3 \%$ for mental arithmetic task and rest. Figure 3 shows the averaged $\mathrm{HbO}$ and standard deviation for mental arithmetic and rest task. Tables 7 and 8 provide the comparison of all classifiers-in terms of average 
TABLE 3: Classification accuracy using $k \mathrm{NN}$ among all subjects.

\begin{tabular}{|c|c|c|c|c|c|c|c|}
\hline Feature combination & S1 & $\mathrm{S} 2$ & S3 & $\mathrm{S} 4$ & S5 & S6 & S7 \\
\hline Mean \& slope & 94.4 & 94.6 & 95.5 & 95.1 & 94.4 & 93.5 & 94.6 \\
\hline Mean \& peak & 95.9 & 96.2 & 97.2 & 97.6 & 97.6 & 97.1 & 96.7 \\
\hline Mean \& variance & 89.1 & 92.6 & 91.5 & 91.3 & 90.7 & 89.6 & 94.0 \\
\hline Slope \& peak & 92.5 & 91.1 & 91.7 & 95.9 & 93.1 & 93.6 & 92.6 \\
\hline Slope \& variance & 95.0 & 95.1 & 94.0 & 93.7 & 93.2 & 92.5 & 93.6 \\
\hline Peak \& variance & 88.2 & 88.7 & 86.8 & 93.5 & 88.6 & 90.2 & 86.3 \\
\hline Peak \& skewness & 64.4 & 66.9 & 64.5 & 65.4 & 62.5 & 58.0 & 61.0 \\
\hline Mean \& skewness & 53.7 & 57.7 & 55.6 & 54.1 & 53.7 & 52.2 & 58.6 \\
\hline Slope \& skewness & 50.8 & 47.8 & 51.4 & 50.1 & 49.9 & 51.3 & 53.8 \\
\hline Kurtosis \& skewness & 47.3 & 50.7 & 55.0 & 51.3 & 54.3 & 60.1 & 54.8 \\
\hline Variance \& skewness & 50.6 & 48.1 & 51.2 & 49.7 & 49.3 & 51.4 & 54.7 \\
\hline Peak \& kurtosis & 65.4 & 63.4 & 59.2 & 65.7 & 62.7 & 55.0 & 60.7 \\
\hline Mean \& kurtosis & 53.5 & 55.0 & 52.9 & 52.1 & 52.2 & 51.3 & 53.7 \\
\hline Slope \& kurtosis & 52.1 & 49.8 & 50.7 & 48.6 & 50.9 & 48.8 & 50.7 \\
\hline Variance \& kurtosis & 51.9 & 50.4 & 50.6 & 48.9 & 50.6 & 48.8 & 50.7 \\
\hline
\end{tabular}

TABLE 4: Classification accuracy using Naïve Bayes among all subjects.

\begin{tabular}{|c|c|c|c|c|c|c|c|}
\hline Feature combination & S1 & S2 & S3 & $\mathrm{S} 4$ & S5 & S6 & S7 \\
\hline Mean \& slope & 95.6 & 96.9 & 95.2 & 96.1 & 96.4 & 94.7 & 96.9 \\
\hline Mean \& peak & 96.5 & 98.1 & 97.1 & 97.9 & 96.0 & 97.9 & 98.0 \\
\hline Mean \& variance & 97.5 & 98.1 & 96.0 & 96.6 & 96.4 & 95.7 & 97.1 \\
\hline Slope \& peak & 94.4 & 95.0 & 89.2 & 95.2 & 95.0 & 93.6 & 96.4 \\
\hline Slope \& variance & 98.0 & 98.0 & 92.0 & 95.2 & 94.9 & 92.0 & 94.4 \\
\hline Peak \& variance & 96.9 & 98.1 & 92.9 & 96.9 & 96.2 & 92.4 & 95.4 \\
\hline Peak \& skewness & 89.6 & 88.6 & 82.8 & 91.8 & 87.2 & 88.0 & 86.0 \\
\hline Mean \& skewness & 89.3 & 91.1 & 89.1 & 90.6 & 89.3 & 87.7 & 93.2 \\
\hline Slope \& skewness & 89.5 & 89.6 & 83.9 & 87.8 & 90.0 & 86.5 & 91.3 \\
\hline Kurtosis \& skewness & 51.1 & 50.8 & 51.1 & 52.7 & 51.8 & 55.8 & 51.9 \\
\hline Variance \& skewness & 97.7 & 97.9 & 90.3 & 95.6 & 94.7 & 90.6 & 92.1 \\
\hline Peak \& kurtosis & 89.6 & 88.2 & 82.8 & 92.0 & 87.1 & 88.2 & 86.2 \\
\hline Mean \& kurtosis & 89.1 & 91.2 & 88.8 & 90.7 & 89.0 & 87.6 & 93.9 \\
\hline Slope \& kurtosis & 89.6 & 89.6 & 83.9 & 87.7 & 89.6 & 85.8 & 91.2 \\
\hline Variance \& kurtosis & 97.6 & 97.9 & 90.5 & 95.4 & 94.5 & 90.6 & 92.0 \\
\hline
\end{tabular}

classification accuracies, precision, and recall-across all subjects for 2- and 3-feature combination, respectively. In order to validate that our ANN classification accuracies were statistically discriminant, we applied Student's $t$-test. The $p$ values obtained using the ANN values versus those of all of the other classifiers were less than 0.05 for all of the $\Delta c_{\mathrm{HbO}}(t)$ signals, thus establishing the statistical significance of ANN's performance.

Several previous studies have used multiple types of classifiers to extract the classification accuracies for fNIRSbased BCI system. For example, Naseer et al. [19] have used
LDA and SVM to acquire the classification accuracies for a two-class BCI system, the classification accuracies were 74.2 and $82.1 \%$ respectively. Moreover, Khan and Hong [72] used LDA and SVM classifiers for a two-class BCI system; the classification accuracies were 84.6 and $85.8 \%$. In the present study, the six different classifiers were used to obtain the highest average classification accuracies for a two-class (metal arithmetic and rest) BCI system. The ANN classifier showed the maximum average classification accuracies 91.4 and $96.3 \%$ for 2 - and 3-dimensional combinations of features derived from $\Delta c_{\mathrm{HbO}}(t)$ signals, respectively. Figure 4 plots the 
TABLE 5: Classification accuracy using SVM among all subjects.

\begin{tabular}{|c|c|c|c|c|c|c|c|}
\hline Feature combination & S1 & S2 & S3 & S4 & S5 & S6 & S7 \\
\hline Mean \& slope & 93.2 & 95.5 & 92.5 & 95.7 & 95.2 & 93.0 & 94.2 \\
\hline Mean \& peak & 97.0 & 98.5 & 98.5 & 98.7 & 97.7 & 98.5 & 98.7 \\
\hline Mean \& variance & 97.7 & 98.0 & 97.7 & 98.0 & 97.7 & 97.0 & 97.2 \\
\hline Slope \& peak & 92.7 & 92.7 & 88.9 & 96.0 & 94.2 & 90.2 & 91.7 \\
\hline Slope \& variance & 98.0 & 98.0 & 97.5 & 97.7 & 96.2 & 95.2 & 96.0 \\
\hline Peak \& variance & 98.0 & 98.7 & 97.5 & 97.5 & 98.0 & 96.7 & 98.5 \\
\hline Peak \& skewness & 92.7 & 85.2 & 83.9 & 93.7 & 90.5 & 86.9 & 83.4 \\
\hline Mean \& skewness & 89.5 & 88.9 & 84.9 & 88.2 & 88.4 & 86.2 & 87.7 \\
\hline Slope \& skewness & 82.4 & 86.4 & 84.9 & 84.7 & 86.4 & 82.7 & 86.7 \\
\hline Kurtosis \& skewness & 54.5 & 54.8 & 51.3 & 52.5 & 54.8 & 52.8 & 50.8 \\
\hline Variance \& skewness & 98.0 & 98.0 & 97.0 & 97.5 & 96.2 & 95.7 & 96.2 \\
\hline Peak \& kurtosis & 90.2 & 83.4 & 81.2 & 94.0 & 86.2 & 84.2 & 83.2 \\
\hline Mean \& kurtosis & 87.7 & 89.2 & 85.4 & 89.2 & 88.9 & 84.4 & 87.7 \\
\hline Slope \& kurtosis & 82.7 & 86.7 & 83.9 & 83.2 & 86.4 & 81.2 & 86.2 \\
\hline Variance \& kurtosis & 98.5 & 98.5 & 97.2 & 97.7 & 96.7 & 95.7 & 96.7 \\
\hline
\end{tabular}

TABLE 6: Classification accuracy using ANN among all subjects.

\begin{tabular}{|c|c|c|c|c|c|c|c|}
\hline Feature combination & S1 & S2 & S3 & S4 & S5 & S6 & S7 \\
\hline Mean \& slope & 95.6 & 97.4 & 94.9 & 97.1 & 96.6 & 96.0 & 95.6 \\
\hline Mean \& peak & 96.9 & 98.5 & 98.1 & 98.7 & 97.9 & 98.4 & 98.4 \\
\hline Mean \& variance & 98.0 & 98.4 & 98.1 & 97.7 & 98.2 & 96.4 & 98.2 \\
\hline Slope \& peak & 95.4 & 92.3 & 95.9 & 96.1 & 95.5 & 90.2 & 92.7 \\
\hline Slope \& variance & 97.9 & 98.4 & 97.7 & 98.2 & 98.0 & 97.0 & 98.0 \\
\hline Peak \& variance & 98.1 & 97.9 & 97.4 & 97.2 & 98.0 & 97.4 & 98.4 \\
\hline Peak \& skewness & 92.8 & 89.0 & 84.2 & 94.1 & 91.6 & 88.5 & 84.7 \\
\hline Mean \& skewness & 92.0 & 94.0 & 90.6 & 92.8 & 89.8 & 88.7 & 92.7 \\
\hline Slope \& skewness & 90.8 & 88.0 & 88.2 & 90.7 & 90.0 & 91.6 & 90.6 \\
\hline Kurtosis \& skewness & 54.5 & 55.7 & 53.6 & 55.6 & 56.2 & 55.7 & 51.6 \\
\hline Variance \& skewness & 98.4 & 98.5 & 97.6 & 98.4 & 97.2 & 97.0 & 98.0 \\
\hline Peak \& kurtosis & 90.1 & 88.5 & 81.4 & 94.1 & 87.8 & 87.5 & 84.9 \\
\hline Mean \& kurtosis & 90.1 & 93.5 & 89.0 & 91.6 & 90.7 & 89.8 & 91.5 \\
\hline Slope \& kurtosis & 91.3 & 88.1 & 88.2 & 90.0 & 90.6 & 91.9 & 90.3 \\
\hline Variance \& kurtosis & 98.4 & 98.4 & 97.1 & 97.4 & 97.6 & 96.6 & 95.5 \\
\hline
\end{tabular}

average accuracies of all of the classifiers used in this study for 2- and 3-dimensional combinations of features derived from $\Delta \mathcal{C}_{\mathrm{HbO}}(t)$ signals. One of the limitations of the current work is the small number of subjects. Analysis with large number of subject can yield to well establishing of the results. Another limitation of our current study is that we used the twoclass mental task (metal arithmetic and rest) for an fNIRSbased BCI system. For three- and more-class BCI problems, other classifier modalities might yield better results. In any case, further research entailing the examination of the results of multiple mental task classifications using different types of classification modalities for fNIRS-based BCI systems is required. Furthermore, inherent delay in fNIRS systems can be removed by detection of initial dips to improve BCI accuracy [73].

\section{Conclusion}

In this study, we examined the effects of using different classification modalities for the classification of a twoclass functional near-infrared spectroscopy- (fNIRS-) based brain-computer interface (BCI) according to a mental arithmetic task and rest experimental paradigm. It was shown 
TABLE 7: Averaged values of the classification accuracies, precisions, and recalls of 2-feature combination across all subjects.

\begin{tabular}{|c|c|c|c|c|c|c|c|c|}
\hline Classifiers & S1 & S2 & S3 & S4 & S5 & S6 & S7 & Average \\
\hline \multicolumn{9}{|l|}{$\mathrm{LDA}$} \\
\hline Accuracy & 72.74 & 72.49 & 70.09 & 73.20 & 71.74 & 70.44 & 70.80 & $71.6 \pm 1.1$ \\
\hline Precision & 79.34 & 79.62 & 79.74 & 68.73 & 67.28 & 66.21 & 66.36 & $72.8 \pm 6.2$ \\
\hline Recall & 66.50 & 65.28 & 58.30 & 83.45 & 80.65 & 81.43 & 78.70 & $73.5 \pm 9.2$ \\
\hline \multicolumn{9}{|l|}{ QDA } \\
\hline Accuracy & 90.78 & 91.57 & 87.49 & 91.12 & 89.98 & 88.83 & 90.57 & $90.1 \pm 1.3$ \\
\hline Precision & 93.80 & 95.84 & 95.32 & 87.68 & 86.27 & 84.00 & 87.28 & $90.0 \pm 4.4$ \\
\hline Recall & 89.63 & 88.80 & 79.53 & 96.50 & 93.65 & 96.82 & 93.65 & $91.2 \pm 5.5$ \\
\hline \multicolumn{9}{|l|}{$k \mathrm{NN}$} \\
\hline Accuracy & 69.63 & 69.87 & 69.85 & 70.19 & 69.58 & 68.89 & 70.44 & $69.8 \pm 0.5$ \\
\hline Precision & 67.97 & 68.73 & 69.90 & 70.31 & 71.24 & 67.16 & 68.18 & $69.1 \pm 1.3$ \\
\hline Recall & 70.81 & 72.14 & 72.31 & 68.21 & 74.53 & 66.32 & 68.38 & $70.4 \pm 2.6$ \\
\hline \multicolumn{9}{|l|}{ Naïve Bayes } \\
\hline Accuracy & 90.79 & 91.27 & 87.04 & 90.82 & 89.86 & 88.47 & 90.39 & $89.8 \pm 1.4$ \\
\hline Precision & 88.52 & 88.01 & 81.73 & 95.69 & 94.88 & 95.93 & 95.48 & $91.5 \pm 5.1$ \\
\hline Recall & 92.12 & 94.16 & 94.76 & 85.61 & 85.96 & 79.90 & 86.85 & $88.5 \pm 5.0$ \\
\hline \multicolumn{9}{|l|}{ SVM } \\
\hline Accuracy & 90.18 & 90.17 & 88.16 & 90.96 & 90.25 & 88.02 & 88.99 & $89.5 \pm 1.0$ \\
\hline Precision & 87.04 & 95.84 & 95.32 & 87.68 & 86.27 & 84.00 & 87.28 & $89.1 \pm 4.2$ \\
\hline Recall & 93.80 & 88.80 & 79.53 & 96.50 & 93.65 & 96.82 & 93.65 & $91.8 \pm 5.5$ \\
\hline \multicolumn{9}{|l|}{ ANN } \\
\hline Accuracy & 92.02 & 91.77 & 90.13 & 92.65 & 91.71 & 90.85 & 90.74 & $91.4 \pm 0.3$ \\
\hline Precision & 94.47 & 89.73 & 93.20 & 90.73 & 86.68 & 88.03 & 87.65 & $90.1 \pm 2.7$ \\
\hline Recall & 88.00 & 86.33 & 85.13 & 95.33 & 94.05 & 95.73 & 95.63 & $91.5 \pm 4.4$ \\
\hline
\end{tabular}

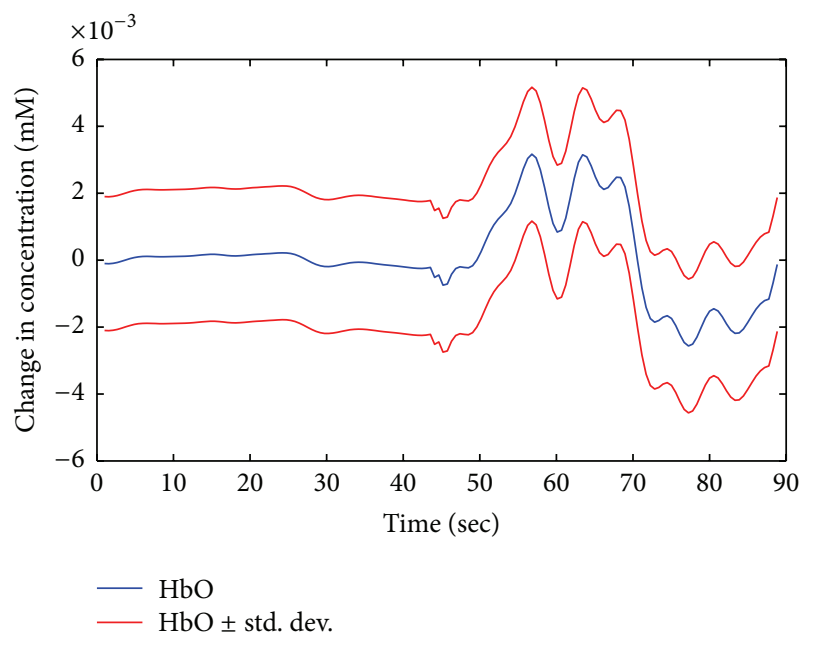

Figure 3: The averaged $\mathrm{HbO}$ and standard deviation (subject 2) for mental arithmetic and rest.

that ANN has the highest classification accuracies among the classification modalities used in this study for both 2and 3-dimensional feature sets derived from $\Delta c_{\mathrm{HbO}}(t)$ signals across seven subjects. The results of this study represent a

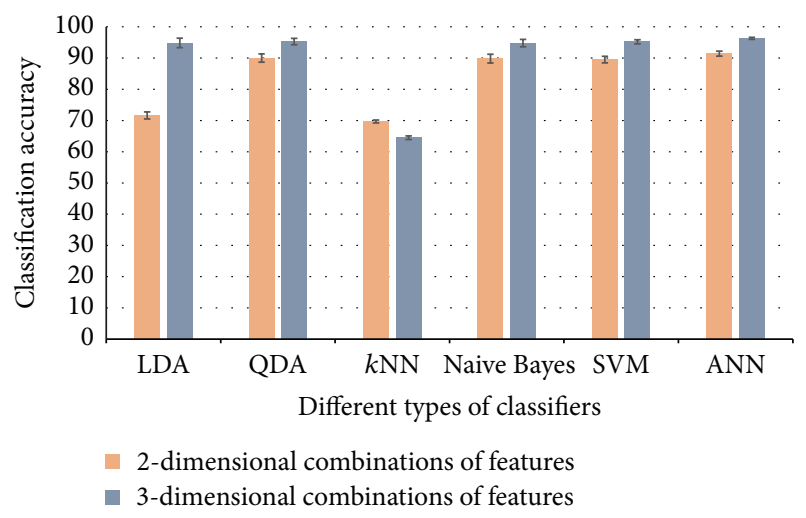

FIGURE 4: Classification accuracies using different types of classifiers from 2- and 3-dimensional combinations of features of $\Delta c_{\mathrm{HbO}}(t)$ signals across all subjects.

significant step forward in the on-going improvement of the classification accuracies of fNIRS-based BCI systems.

\section{Competing Interests}

The authors declare that there are no competing interests regarding the publication of this paper. 
TABLE 8: Averaged values of the classification accuracies, precisions, and recalls of 3-feature combinations across all subjects.

\begin{tabular}{|c|c|c|c|c|c|c|c|c|}
\hline Classifiers & S1 & S2 & S3 & S4 & S5 & S6 & S7 & Average \\
\hline \multicolumn{9}{|l|}{ LDA } \\
\hline Accuracy & 81.24 & 81.76 & 78.41 & 80.54 & 79.68 & 77.88 & 77.78 & $79.6 \pm 1.5$ \\
\hline Precision & 89.9 & 89.21 & 89.66 & 75.09 & 74.21 & 72.37 & 72.53 & $80.4 \pm 7.8$ \\
\hline Recall & 73.94 & 73.98 & 65.79 & 91.33 & 90.09 & 89.88 & 87.48 & $81.8 \pm 9.5$ \\
\hline \multicolumn{9}{|l|}{ QDA } \\
\hline Accuracy & 95.84 & 96.58 & 93.49 & 95.96 & 95.11 & 94.12 & 95.93 & $95.2 \pm 1$ \\
\hline Precision & 97.26 & 98.67 & 98.67 & 93.79 & 92.34 & 90.8 & 93.85 & $95.1 \pm 2.9$ \\
\hline Recall & 94.41 & 94.1 & 88.19 & 98.71 & 98.64 & 98.8 & 98.66 & $95.9 \pm 3.7$ \\
\hline \multicolumn{9}{|l|}{$k \mathrm{NN}$} \\
\hline Accuracy & 63.92 & 65.32 & 64.85 & 64.78 & 64.59 & 63.55 & 65.11 & $64.5 \pm 0.3$ \\
\hline Precision & 63.22 & 62.21 & 63.85 & 64.13 & 65.85 & 61.04 & 61.07 & $63.1 \pm 1.6$ \\
\hline Recall & 65.28 & 67.56 & 66.15 & 61.67 & 69.49 & 60.13 & 61.54 & $64.6 \pm 3.2$ \\
\hline \multicolumn{9}{|l|}{ Naïve Bayes } \\
\hline Accuracy & 95.58 & 96.34 & 92.77 & 95.53 & 94.72 & 93.47 & 95.55 & $94.8 \pm 1.2$ \\
\hline Precision & 94.45 & 94.22 & 88.46 & 98.73 & 98.35 & 98.64 & 98.68 & $95.9 \pm 3.6$ \\
\hline Recall & 96.98 & 99.12 & 98.95 & 92.22 & 90.89 & 88.1 & 92.29 & $94.1 \pm 3.9$ \\
\hline \multicolumn{9}{|l|}{ SVM } \\
\hline Accuracy & 95.79 & 95.75 & 94.21 & 95.97 & 95.58 & 94.47 & 94.85 & $95.2 \pm 0.7$ \\
\hline Precision & 93.22 & 99.03 & 98.67 & 93.79 & 92.34 & 90.8 & 93.85 & $94.5 \pm 2.9$ \\
\hline Recall & 99.12 & 94.1 & 88.19 & 98.71 & 98.64 & 98.8 & 98.66 & $96.6 \pm 3.8$ \\
\hline \multicolumn{9}{|l|}{$\mathrm{ANN}$} \\
\hline Accuracy & 96.48 & 96.49 & 95.78 & 96.87 & 96.4 & 95.97 & 96.41 & $96.3 \pm 0.3$ \\
\hline Precision & 93.9 & 94.4 & 98.1 & 95.05 & 93.26 & 91.59 & 93.71 & $94.3 \pm 1.9$ \\
\hline Recall & 91.9 & 92.8 & 91.55 & 93.64 & 98.39 & 98.46 & 98.67 & $95.1 \pm 3.0$ \\
\hline
\end{tabular}

\section{Acknowledgments}

This study was supported by the National Research Foundation of Korea under the auspices of the Ministry of Science, ICT and Future Planning, Korea (Grant no. NRF2014R1A2A1A10049727) and the Higher Education Commission (HEC) of Pakistan (Grant no. SRGP-726).

\section{References}

[1] J. R. Wolpaw, N. Birbaumer, D. J. McFarland, G. Pfurtscheller, and T. M. Vaughan, "Brain-computer interfaces for communication and control," Clinical Neurophysiology, vol. 113, no. 6, pp. 767-791, 2002.

[2] J. Viventi, D.-H. Kim, L. Vigeland et al., "Flexible, foldable, actively multiplexed, high-density electrode array for mapping brain activity in vivo," Nature Neuroscience, vol. 14, no. 12, pp. 1599-1605, 2011.

[3] B. A. Wester, R. H. Lee, and M. C. LaPlaca, "Development and characterization of in vivo flexible electrodes compatible with large tissue displacements," Journal of Neural Engineering, vol. 6, no. 2, Article ID 024002, 2009.

[4] S. Thongpang, T. J. Richner, S. K. Brodnick et al., "A microelectrocorticography platform and deployment strategies for chronic BCI applications," Clinical EEG and Neuroscience, vol. 42, no. 4, pp. 259-265, 2011.

[5] M. Salvaris and F. Sepulveda, "Classification effects of real and imaginary movement selective attention tasks on a P300-based brain-computer interface," Journal of Neural Engineering, vol. 7, no. 5, Article ID 056004, 2010.

[6] K. Choi, "Electroencephalography (EEG)-based neurofeedback training for brain-computer interface (BCI)," Experimental Brain Research, vol. 231, no. 3, pp. 351-365, 2013.

[7] M. J. Khan, M. J. Hong, and K.-S. Hong, "Decoding of four movement directions using hybrid NIRS-EEG brain-computer interface," Frontiers in Human Neuroscience, vol. 8, no. 1, article 244, 2014.

[8] I. Rejer, "Genetic algorithm with aggressive mutation for feature selection in BCI feature space," Pattern Analysis and Applications, vol. 18, no. 3, pp. 485-492, 2015.

[9] W.-Y. Hsu, "Improving classification accuracy of motor imagery EEG using genetic feature selection," Clinical EEG and Neuroscience, vol. 45, no. 3, pp. 163-168, 2014.

[10] B. Sorger, B. Dahmen, J. Reithler et al., "Another kind of 'BOLD Response': answering multiple-choice questions via online decoded single-trial brain signals," Progress in Brain Research, vol. 177, pp. 275-292, 2009.

[11] C. Enzinger, S. Ropele, F. Fazekas et al., "Brain motor system function in a patient with complete spinal cord injury following extensive brain-computer interface training," Experimental Brain Research, vol. 190, no. 2, pp. 215-223, 2008.

[12] A. M. Mendrik, K. L. Vincken, H. J. Kuijf et al., "MRBrainS challenge: online evaluation framework for brain image segmentation in 3T MRI scans," Computational Intelligence and Neuroscience, vol. 2015, Article ID 813696, 16 pages, 2015.

[13] M. Ferrari, I. Giannini, G. Sideri, and E. Zanette, "Continuous non invasive monitoring of human brain by near infrared 
spectroscopy," Advances in Experimental Medicine and Biology, vol. 191, pp. 873-882, 1985.

[14] T. Kato, A. Kamei, S. Takashima, and T. Ozaki, "Human visual cortical function during photic stimulation monitoring by means of near-infrared spectroscopy," Journal of Cerebral Blood Flow and Metabolism, vol. 13, no. 3, pp. 516-520, 1993.

[15] S. M. Coyle, T. E. Ward, C. M. Markham, and G. McDarby, "On the suitability of near-infrared (NIR) systems for nextgeneration brain-computer interfaces," Physiological Measurement, vol. 25, no. 4, article 815, 2004.

[16] S. M. Coyle, T. E. Ward, and C. M. Markham, "Braincomputer interface using a simplified functional near-infrared spectroscopy system," Journal of Neural Engineering, vol. 4, no. 3, article 219, 2007.

[17] M. Naito, Y. Michioka, K. Ozawa, Y. Ito, M. Kiguchi, and T. Kanazawa, "A communication means for totally locked-in ALS patients based on changes in cerebral blood volume measured with near-infrared light," IEICE Transactions on Information and Systems, vol. 90, no. 7, pp. 1028-1037, 2007.

[18] N. Naseer and K.-S. Hong, "Classification of functional nearinfrared spectroscopy signals corresponding to the right- and left-wrist motor imagery for development of a brain-computer interface," Neuroscience Letters, vol. 553, pp. 84-89, 2013.

[19] N. Naseer, M. J. Hong, and K.-S. Hong, "Online binary decision decoding using functional near-infrared spectroscopy for the development of brain-computer interface," Experimental Brain Research, vol. 232, no. 2, pp. 555-564, 2014.

[20] K.-S. Hong and H.-D. Nguyen, "State-space models of impulse hemodynamic responses over motor, somatosensory, and visual cortices," Biomedical Optics Express, vol. 5, no. 6, pp. 1778-1798, 2014.

[21] N. Naseer, F. M. Noori, N. K. Qureshi, and K. Hong, "Determining optimal feature-combination for LDA classification of functional near-infrared spectroscopy signals in brain-computer interface application," Frontiers in Human Neuroscience, vol. 10, article 237, 2016.

[22] A. K. Vellage, M. Veit, X. Kobeleva, S. Petri, S. Vielhaber, and N. G. Müller, "Working memory network changes in ALS: an fMRI Study," Frontiers in Neuroscience, vol. 10, article 158, 2016.

[23] N. Naseer and K.-S. Hong, "fNIRS-based brain-computer interfaces: a review," Frontiers in Human Neuroscience, vol. 9, article 3, 2015.

[24] A. Villringer, J. Planck, C. Hock, L. Schleinkofer, and U. Dirnagl, "Near infrared spectroscopy (NIRS): a new tool to study hemodynamic changes during activation of brain function in human adults," Neuroscience Letters, vol. 154, no. 1-2, pp. 101104, 1993.

[25] Y. Hoshi, H. Onoe, Y. Watanabe et al., "Non-synchronous behavior of neuronal activity, oxidative metabolism and blood supply during mental tasks in man," Neuroscience Letters, vol. 172, no. 1-2, pp. 129-133, 1994.

[26] Y. Hoshi and M. Tamura, "Near-infrared optical detection of sequential brain activation in the prefrontal cortex during mental tasks," NeuroImage, vol. 5, no. 4, pp. 292-297, 1997.

[27] D. T. Delpy, M. Cope, P. van der Zee, S. Arridge, S. Wray, and J. Wyatt, "Estimation of optical path length through tissue from direct time of flight measurement," Physics in Medicine and Biology, vol. 33, no. 12, pp. 1433-1442, 1988.

[28] A. Sassaroli and S. Fantini, "Comment on the modified beerlambert law for scattering media," Physics in Medicine and Biology, vol. 49, no. 14, pp. N255-N257, 2004.
[29] F. F. Jobsis, "Noninvasive, infrared monitoring of cerebral and myocardial oxygen sufficiency and circulatory parameters," Science, vol. 198, no. 4323, pp. 1264-1267, 1977.

[30] S. D. Power, T. H. Falk, and T. Chau, "Classification of prefrontal activity due to mental arithmetic and music imagery using hidden Markov models and frequency domain near-infrared spectroscopy," Journal of Neural Engineering, vol. 7, no. 2, Article ID 026002, 2010.

[31] T. H. Falk, M. Guirgis, S. Power, and T. T. Chau, “Taking NIRSBCIs outside the lab: towards achieving robustness against environment noise," IEEE Transactions on Neural Systems and Rehabilitation Engineering, vol. 19, no. 2, pp. 136-146, 2011.

[32] H. Santosa, M. J. Hong, and K.-S. Hong, "Lateralization of music processing with noises in the auditory cortex: an fNIRS study," Frontiers in Behavioral Neuroscience, vol. 8, article 418, 2014.

[33] G. Bauernfeind, R. Scherer, G. Pfurtscheller, and C. Neuper, "Single-trial classification of antagonistic oxyhemoglobin responses during mental arithmetic," Medical and Biological Engineering and Computing, vol. 49, no. 9, pp. 979-984, 2011.

[34] B. Abibullaev and J. An, "Classification of frontal cortex haemodynamic responses during cognitive tasks using wavelet transforms and machine learning algorithms," Medical Engineering \& Physics, vol. 34, no. 10, pp. 1394-1410, 2012.

[35] B. Abibullaev, J. An, and J.-I. Moon, "Neural network classification of brain hemodynamic responses from four mental tasks," International Journal of Optomechatronics, vol. 5, no. 4, pp. 340359, 2011.

[36] A. Faress and T. Chau, "Towards a multimodal brain-computer interface: combining fNIRS and fTCD measurements to enable higher classification accuracy," NeuroImage, vol. 77, pp. 186-194, 2013.

[37] H.-J. Hwang, J.-H. Lim, D.-W. Kim, and C.-H. Im, "Evaluation of various mental task combinations for near-infrared spectroscopy-based brain-computer interfaces," Journal of Biomedical Optics, vol. 19, no. 7, Article ID 077005, 2014.

[38] H. Ayaz, P. A. Shewokis, S. Bunce, K. Izzetoglu, B. Willems, and B. Onaral, "Optical brain monitoring for operator training and mental workload assessment," NeuroImage, vol. 59, no. 1, pp. 3647, 2012.

[39] H. Ayaz, P. Shewokis, S. Bunce, M. Schultheis, and B. Onaral, "Assessment of cognitive neural correlates for a functional near infrared-based brain-computer interface system," in Foundations of Augmented Cognition. Neuroergonomics and Operational Neuroscience, vol. 5638, pp. 699-708, Springer, Berlin, Germany, 2009.

[40] T. Gateau, G. Durantin, F. Lancelot, S. Scannella, and F. Dehais, "Real-time state estimation in a flight simulator using fNIRS," PLoS ONE, vol. 10, no. 3, Article ID e0121279, 2015.

[41] H. Matsuyama, H. Asama, and M. Otake, "Design of differential near-infrared spectroscopy based brain machine interface," in Proceedings of the 18th IEEE International Symposium Robot and Human Interactive Communication (IEEE RO-MAN), pp. 775780, 2009.

[42] H. Santosa, M. J. Hong, S.-P. Kim, and K.-S. Hong, "Noise reduction in functional near-infrared spectroscopy signals by independent component analysis," Review of Scientific Instruments, vol. 84, no. 7, Article ID 073106, 2013.

[43] S. D. Power, A. Kushki, and T. Chau, "Towards a systempaced near-infrared spectroscopy brain-computer interface: differentiating prefrontal activity due to mental arithmetic and mental singing from the no-control state," Journal of Neural Engineering, vol. 8, no. 6, Article ID 066004, 2011. 
[44] R. Sitaram, H. Zhang, C. Guan et al., "Temporal classification of multichannel near-infrared spectroscopy signals of motor imagery for developing a brain-computer interface," NeuroImage, vol. 34, no. 4, pp. 1416-1427, 2007.

[45] L. Holper and M. Wolf, "Single-trial classification of motor imagery differing in task complexity: a functional near-infrared spectroscopy study," Journal of NeuroEngineering and Rehabilitation, vol. 8, no. 1, article 34, 2011.

[46] S. Luu and T. Chau, "Decoding subjective preference from single-trial near-infrared spectroscopy signals," Journal of Neural Engineering, vol. 6, no. 1, Article ID 016003, 2009.

[47] H. Sato, Y. Fuchino, M. Kiguchi et al., "Intersubject variability of near-infrared spectroscopy signals during sensorimotor cortex activation," Journal of Biomedical Optics, vol. 10, no. 4, Article ID 044001, 2005.

[48] G. Jasdzewski, G. Strangman, J. Wagner, K. K. Kwong, R. A. Poldrack, and D. A. Boas, "Differences in the hemodynamic response to event-related motor and visual paradigms as measured by near-infrared spectroscopy," NeuroImage, vol. 20, no. 1, pp. 479-488, 2003.

[49] X.-S. Hu, K.-S. Hong, S. S. Ge, and M.-Y. Jeong, "Kalman estimator- and general linear model-based on-line brain activation mapping by near-infrared spectroscopy," BioMedical Engineering Online, vol. 9, article 82, 15 pages, 2010.

[50] X.-S. Hu, K.-S. Hong, and S. S. Ge, "Recognition of stimulusevoked neuronal optical response by identifying chaos levels of near-infrared spectroscopy time series," Neuroscience Letters, vol. 504, no. 2, pp. 115-120, 2011.

[51] X.-S. Hu, K.-S. Hong, and S. S. Ge, "Reduction of trial-to-trial variability in functional near-infrared spectroscopy signals by accounting for resting-state functional connectivity," Journal of Biomedical Optics, vol. 18, no. 1, Article ID 017003, pp. 1-9, 2013.

[52] N. Naseer and K.-S. Hong, "Decoding answers to four-choice questions using functional near infrared spectroscopy," Journal of Near Infrared Spectroscopy, vol. 23, no. 1, pp. 23-31, 2015.

[53] P. W. McCormick, M. Stewart, G. Lewis, M. Dujovny, and J. I. Ausman, "Intracerebral penetration of infrared light: technical note," Journal of Neurosurgery, vol. 76, no. 2, pp. 315-318, 1992.

[54] G. Gratton, C. R. Brumback, B. A. Gordon, M. A. Pearson, K. A. Low, and M. Fabiani, "Effects of measurement method, wavelength, and source-detector distance on the fast optical signal," NeuroImage, vol. 32, no. 4, pp. 1576-1590, 2006.

[55] K.-S. Hong, N. Naseer, and Y.-H. Kim, "Classification of prefrontal and motor cortex signals for three-class fNIRS-BCI," Neuroscience Letters, vol. 587, pp. 87-92, 2015.

[56] S. D. Power and T. Chau, "Automatic single-trial classification of prefrontal hemodynamic activity in an individual with Duchenne muscular dystrophy," Developmental Neurorehabilitation, vol. 16, no. 1, pp. 67-72, 2013.

[57] X. Cui, S. Bray, and A. L. Reiss, "Speeded near infrared spectroscopy (NIRS) response detection," PLoS ONE, vol. 5, no. 11, Article ID e15474, 2010.

[58] K.-S. Hong and H. Santosa, "Decoding four different soundcategories in the auditory cortex using functional near-infrared spectroscopy," Hearing Research, vol. 333, pp. 157-166, 2016.

[59] K. Tai and T. Chau, "Single-trial classification of NIRS signals during emotional induction tasks: towards a corporeal machine interface," Journal of NeuroEngineering and Rehabilitation, vol. 6, no. 1, article 39, 2009.
[60] F. Lotte, M. Congedo, A. Lécuyer, F. Lamarche, and B. Arnaldi, "A review of classification algorithms for EEG-based braincomputer interfaces," Journal of Neural Engineering, vol. 4, no. 2, pp. R1-R13, 2007.

[61] S. Moghimi, A. Kushki, S. Power, A. M. Guerguerian, and T. Chau, "Automatic detection of a prefrontal cortical response to emotionally rated music using multi-channel near-infrared spectroscopy," Journal of Neural Engineering, vol. 9, no. 2, Article ID 026022, 2012.

[62] M. R. Bhutta, M. J. Hong, Y.-H. Kim, and K.-S. Hong, "Singletrial lie detection using a combined fNIRS-polygraph system," Frontiers in Psychology, vol. 6, article 709, 2015.

[63] J. H. Friedman, "Regularized discriminant analysis," Journal of the American Statistical Association, vol. 84, no. 405, pp. 165-175, 1989.

[64] T. Cover and P. Hart, "Nearest neighbor pattern classification," IEEE Transactions on Information Theory, vol. 13, no. 1, pp. 2127, 1967.

[65] D. Geiger, M. Goldszmidt, G. Provan, P. Langley, and P. Smyth, "Bayesian network classifiers," Machine Learning, vol. 29, pp. 131-163, 1997.

[66] J. Shin and J. Jeong, "Multiclass classification of hemodynamic responses for performance improvement of functional nearinfrared spectroscopy-based brain-computer interface," Journal of Biomedical Optics, vol. 19, no. 6, Article ID 067009, 2014.

[67] C. J. C. Burges, "A tutorial on support vector machines for pattern recognition," Data Mining and Knowledge Discovery, vol. 2, no. 2, pp. 121-167, 1998.

[68] X.-S. Hu, K.-S. Hong, and S. S. Ge, "fNIRS-based online deception decoding," Journal of Neural Engineering, vol. 9, no. 2, Article ID 026012, 2012.

[69] N. T. Hai, N. Q. Cuong, T. Q. Dang Khoa, and V. Van Toi, “Temporal hemodynamic classification of two hands tapping using functional near-infrared spectroscopy," Frontiers in Human Neuroscience, vol. 7, article 516, 2013.

[70] J. Chan, S. Power, and T. Chau, "Investigating the need for modelling temporal dependencies in a brain-computer interface with real-time feedback based on near infrared spectra," Journal of Near Infrared Spectroscopy, vol. 20, no. 1, pp. 107-116, 2012.

[71] R. B. Rao, G. Fung, and R. Rosales, "On the dangers of crossvalidation. An experimental evaluation," in SDM, pp. 588-596, 2008.

[72] M. J. Khan and K.-S. Hong, "Passive BCI based on drowsiness detection: an fNIRS study," Biomedical Optics Express, vol. 6, no. 10, pp. 4063-4078, 2015.

[73] K.-S. Hong and N. Naseer, "Reduction of delay in detecting initial dips from functional near-infrared spectroscopy signals using vector-based phase analysis," International Journal of Neural Systems, vol. 26, no. 3, Article ID 1650012, 2016. 

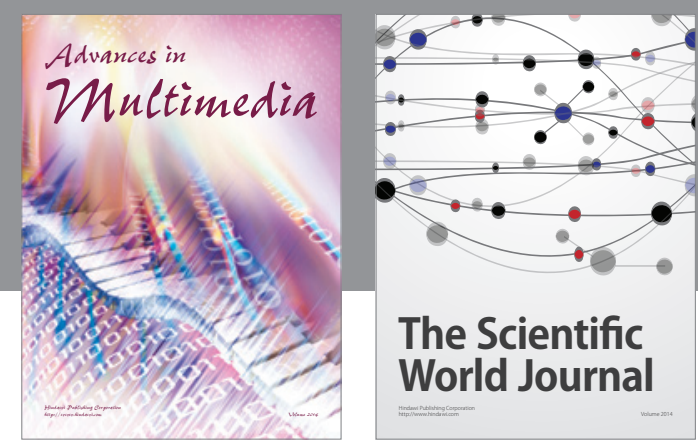

The Scientific World Journal
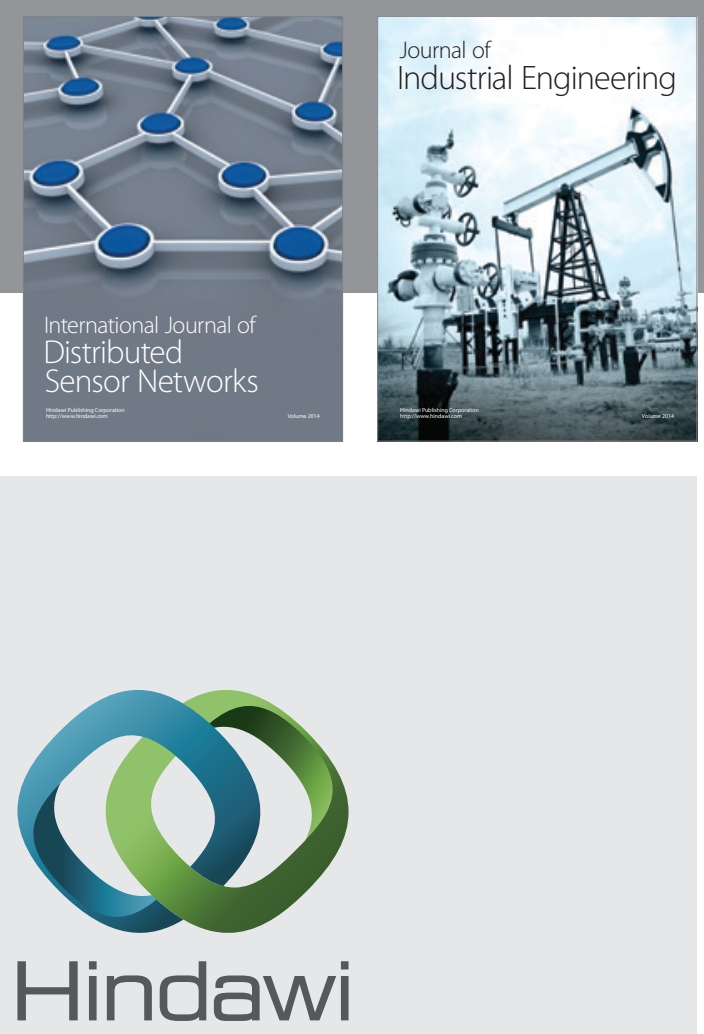

Submit your manuscripts at

http://www.hindawi.com

\section{Computer Networks} and Communications
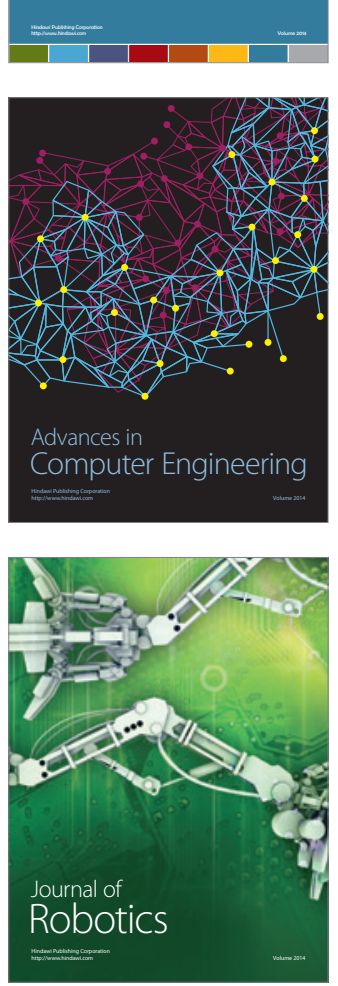
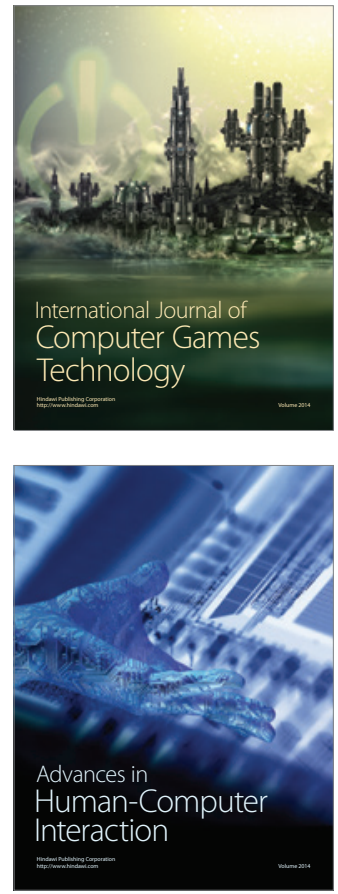
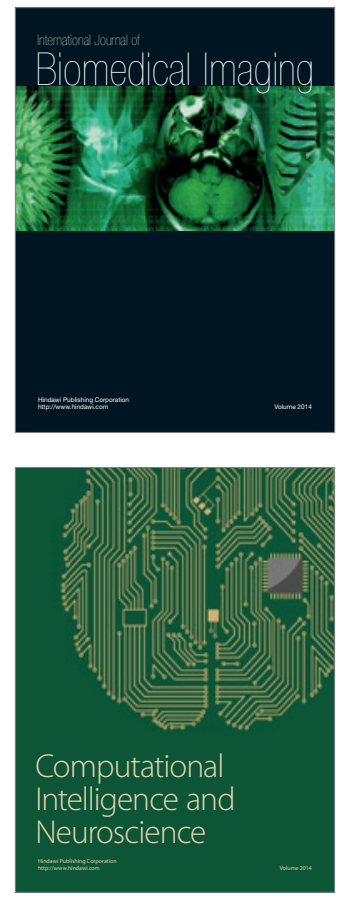
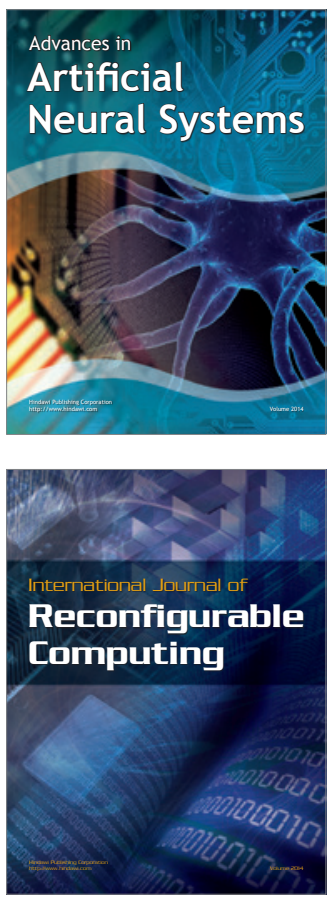
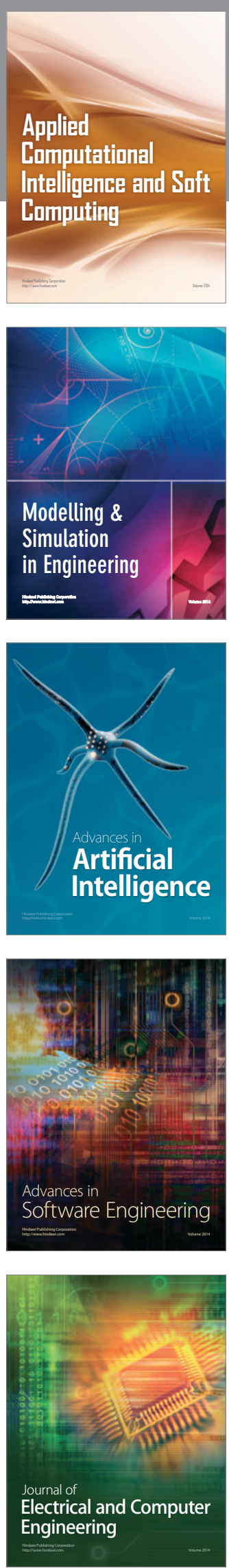